\title{
Stress Energy Tensor Study in Fluid Mechanics
}

Roman Baudrimont

Independent researcher

RomanBaudrimont.cd@gmail.com

\begin{abstract}
Abstrat:This paper is to summarize the involvement of the stress energy tensor in the study of fluid mechanics. In the first part we will see the implication that carries the stress energy tensor in the framework of general relativity. In the second part, we will study the stress energy tensor under the mechanics of perfect fluids, allowing us to lead third party in the case of Newtonian fluids, and in the last part we will see that it is possible to define space-time as a no-Newtonian fluids.
\end{abstract}

Keys word: Fluid Mechanic, Pulse Energy Tensor, General Relativity

\section{Part 1 - The stress energy tensor in general relativity.}

\section{Part A - The stress energy tensor as stress tensor.}

The stress energy tensor is a tensor used in Einstein's equation, to describe the content in energy of spacetime. Indeed, it discloses that the energy and momentum associated with the matter, or any other form of non-gravitational field ${ }^{[1]}$. The stress energy tensor no contain all the information on the retail miscroscopique constituents of matter. For this, thermodynamics and statistical mechanics are necessary.

In a general context, the vector energy flow of the matter is measured by the following equation:

$$
\vec{p}=p^{i} \vec{e}_{i}
$$

Where $\vec{e}_{i}$ is an orthonormal basis. At the same time, according to the source ${ }^{[1]}$, then we can express the vector energy flow of matter and energy per unit of time:

$$
\begin{gathered}
\varphi^{i}=-c T\left(\vec{u}_{0}, \vec{e}_{i}\right) \\
\frac{d E}{d t}=\vec{\varphi} \cdot \vec{n} d S
\end{gathered}
$$

With a tensor field noted $T, \vec{n}$ a normal surface element and area $d S$. It follows that the writing of the stress tensor is then:

$$
S_{i j}=T\left(\vec{e}_{i}, \vec{e}_{j}\right)
$$

The tensor described below is the force exerted by the matter in the direction of $\vec{e}_{i}$ on the unit area $\vec{e}_{j}$ which is normal.

Indeed, the stress tensor is a pressure tensor: it describes what way the matter has a "force" on a "surface", called spacetime.

This interpretation of the stress energy tensor as a stress tensor will be very important for what follows. Before leaving, note that we can write this:

$$
\vec{\varphi}=c^{2} \vec{p}
$$


This equation defines equality between the vector of energy flow and the vector of pulse density to the matter with a factor $c^{2}$.

Therefore, this equation is in fact a kind of mathematical consequence of the mass-energy equivalence: here there is an equivalence between energy flow and a pulse density vector to the matter.

\section{Part B - The stress energy tensor in the Einstein equation.}

General relativity ${ }^{[1]}$ is a theory of gravitation by Einstein written between 1907 and 1915 . It states that the gravitational attraction previously known by Newton's equation is actually a distortion of space-time caused by concentrations of energy. It is described in a simplified way, by this equation:

Or, completely, knowing that:

$$
G_{i j}=\frac{8 \pi G}{c^{4}} T_{i j}
$$

$$
G_{i j}=R_{i j}-\frac{1}{2} G_{i j} R
$$

We have :

$$
R_{i j}-\frac{1}{2} G_{i j} R=\frac{8 \pi G}{c^{4}} T_{i j}
$$

This is a tensor equation. $8 \pi G / c^{\wedge} 4$ is a constant, the tensor $T_{i j}$ is the relativistic stress energy tensor (a matrix $4 \times 4$ ) derived from the flow moments tensor (a matrix $3 \times 3$ ). We find the Einstein tensor $G_{i j}$ and the Ricci curvature tensor $R_{i j}$ and the scalar noted $R$. Finally, there is the metric tensor noted $g_{i j}$.

The tensor $T_{i j}$ can be written with the proper time $\varepsilon$ of special relativity:

$$
d \varepsilon=\frac{d s}{c}=\frac{\sqrt{g_{i j} d x^{i} d x^{j}}}{c}
$$

We can then write the equation, with $\rho$ a density:

$$
T_{i j}=\rho \frac{d x_{i}}{d \varepsilon} \frac{d x_{j}}{d \varepsilon}=\rho c^{2} \frac{d x_{i}}{d s} \frac{d x_{j}}{d s}
$$

Multiplying by the controvarian metric tensor, the energy density can be achieved:

$$
g^{i j} T_{i j}=g^{i j} \rho c^{2} \frac{d x_{i}}{d s} \frac{d x_{j}}{d s}=\rho c^{2}
$$

It is possible to derive the equations of Einstein the principle of least action, the first being the work of Einstein-Hilbert noted $S_{H}$, and the second action to the matter, noted $S_{M}$ :

$$
\begin{aligned}
& S_{H}=\int d^{4} x \sqrt{|g|} R \\
& S_{M}=\int d^{4} x \sqrt{|g|} \varrho
\end{aligned}
$$




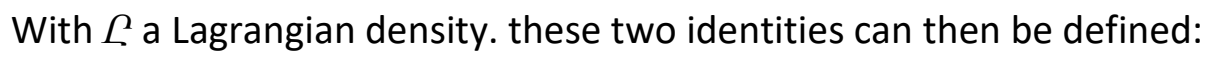

$$
\begin{aligned}
& G_{i j}=-\frac{1}{\sqrt{|g|}} \frac{\delta S_{H}}{\delta g_{i j}} \\
& T_{i j}=-\frac{1}{\sqrt{|g|}} \frac{\delta S_{M}}{\delta g^{i j}}
\end{aligned}
$$

Finally, the stress energy tensor respects the principle of local energy conservation:

Now further analyze the tensor $T_{i j}$.

$$
\nabla^{j} T_{i j}=0
$$

The stress energy tensor in its matrix form, can be written as ${ }^{[2]}$ :

$$
T_{i j}=\left[\begin{array}{cccc}
T_{00} & T_{01} & T_{02} & T_{03} \\
T_{10} & T_{11} & T_{12} & T_{13} \\
T_{20} & T_{21} & T_{22} & T_{23} \\
T_{30} & T_{31} & T_{32} & T_{33}
\end{array}\right]=\left[\begin{array}{cccc}
\rho \gamma^{2} c^{2} & \rho \gamma^{2} c v_{x} & \rho \gamma^{2} c v_{x} & \rho \gamma^{2} c v_{x} \\
\rho \gamma^{2} c v_{x} & \rho \gamma^{2} v_{x} v_{x} & \rho \gamma^{2} v_{x} v_{y} & \rho \gamma^{2} v_{x} v_{z} \\
\rho \gamma^{2} c v_{x} & \rho \gamma^{2} v_{y} v_{x} & \rho \gamma^{2} v_{y} v_{y} & \rho \gamma^{2} v_{y} v_{z} \\
\rho \gamma^{2} c v_{x} & \rho \gamma^{2} v_{z} v_{x} & \rho \gamma^{2} v_{z} v_{y} & \rho \gamma^{2} v_{z} v_{z}
\end{array}\right]
$$

In this tensor include:

- Energy density: $T_{00}$

- Pressure: $T_{11}, T_{12}, T_{13}$

- $\quad$ The flow of energy: $T_{01}, T_{02}, T_{03}$

- Pulse Density: $T_{10}, T_{20}, T_{30}$

- The viscosity: $T_{21}, T_{31}, T_{32}, T_{12}, T_{13}, T_{23}$

The stress energy tensor itself is not easy to understand. First recall that $T_{i j}$ is the flow of the component of the $i$ four-momentum $x_{j}$ we can note the pulse $m u_{i}$ and flow $n u_{j}$. The pulse flow is then noted:

$$
m u_{i} \cdot n u_{j}=\rho u_{i} u_{j}
$$

The analysis of the stress energy tensor is the following.

In the first "line" of the matrix $T_{00}, T_{01}, T_{02}, T_{03}$.

$T_{00}$ is the energy density.

$T_{01}, T_{02}, T_{03}$ corresond the energy flow, as said above. To be more specific, it is the flow of the fourth component, energy, ranging in three directions (direction 1 for example $T_{01}$ ).

Same for the impulse: it has a density and a flow going in three directions $\left(T_{10}, T_{20}, T_{30}\right)$.

In general, the first index indicates what we mean $\left(E, p_{x}, p_{y}, p_{z}\right)$ and the second index indicates the direction of flow $(t, x, y, z)$

Note that in the case of low speeds, that is to say $\gamma=1$, we have then:

$$
T_{i j}=\left[\begin{array}{cccc}
\rho c^{2} & \rho c v_{x} & \rho c v_{y} & \rho c v_{z} \\
\rho c v_{x} & \rho v_{x} v_{x} & \rho v_{x} v_{y} & \rho v_{x} v_{z} \\
\rho c v_{y} & \rho v_{y} v_{x} & \rho v_{y} v_{y} & \rho v_{y} v_{z} \\
\rho c v_{z} & \rho v_{z} v_{x} & \rho v_{z} v_{y} & \rho v_{z} v_{z}
\end{array}\right]=\left[\begin{array}{cccc}
\rho c^{2} & \rho c v_{x} & \rho c v_{y} & \rho c v_{z} \\
\rho c v_{x} & P_{x x} & \tau_{x y} & \tau_{x z} \\
\rho c v_{y} & \tau_{y x} & P_{y y} & \tau_{y z} \\
\rho c v_{z} & \tau_{z x} & \tau_{z y} & P_{z z}
\end{array}\right]
$$

Note while the $T_{21}, T_{31}, T_{32}, T_{12}, T_{13}, T_{23}$ noted by the various $\tau$ correspond in fact at the shear viscosity. 


\section{Part 2 - The stress energy tensor in perfect fluid.}

As I said above, the stress energy tensor of Einstein contains the stress tensor a $3 \times 3$ matrix Thus, we have:

$$
t_{i j}=\left[\begin{array}{lll}
T_{11} & T_{12} & T_{13} \\
T_{21} & T_{22} & T_{23} \\
T_{31} & T_{32} & T_{33}
\end{array}\right]
$$

But for this part, we do not study this tensor. Indeed, the stress energy tensor of an ideal fluid is a tensor not taking into account the viscosity, that is to say against the following elements: $T_{21}, T_{31}, T_{32}, T_{12}, T_{13}, T_{23}$. Moreover, it does not take into account the flow of energy or the pulse density, which reduces us to the following matrix:

$$
T_{i j}=\left[\begin{array}{cccc}
T_{00} & 0 & 0 & 0 \\
0 & T_{11} & 0 & 0 \\
0 & 0 & T_{22} & 0 \\
0 & 0 & 0 & T_{33}
\end{array}\right]=\left[\begin{array}{cccc}
\rho \gamma^{2} c^{2} & 0 & 0 & 0 \\
0 & \rho \gamma^{2} u_{x} u_{x} & 0 & 0 \\
0 & 0 & \rho \gamma^{2} u_{y} u_{y} & 0 \\
0 & 0 & 0 & \rho \gamma^{2} u_{z} u_{z}
\end{array}\right]
$$

In this tensor include:

- Energy density: $T_{00}$

- Pressure: $T_{11}, T_{12}, T_{13}$

Finally, the stress energy tensor becomes a diagonal matrix, computable by its trace:

$$
T_{i j}=\operatorname{Tr}\left[\begin{array}{cccc}
\rho \gamma^{2} c^{2} & 0 & 0 & 0 \\
0 & \rho \gamma^{2} u_{x} u_{x} & 0 & 0 \\
0 & 0 & \rho \gamma^{2} u_{y} u_{y} & 0 \\
0 & 0 & 0 & \rho \gamma^{2} u_{z} u_{z}
\end{array}\right]
$$

And so, in the low speed setting where $\gamma \approx 1$ :

$$
T_{i j}=\operatorname{Tr}\left[\begin{array}{cccc}
\rho c^{2} & 0 & 0 & 0 \\
0 & \rho u_{x} u_{x} & 0 & 0 \\
0 & 0 & \rho u_{y} u_{y} & 0 \\
0 & 0 & 0 & \rho u_{z} u_{z}
\end{array}\right]=\operatorname{Tr}\left[\begin{array}{cccc}
\rho c^{2} & 0 & 0 & 0 \\
0 & P_{x x} & 0 & 0 \\
0 & 0 & P_{y y} & 0 \\
0 & 0 & 0 & P_{z z}
\end{array}\right]
$$

The stress energy tensor can be written as ${ }^{[3]}$ :

$$
T_{i j}=\left(\frac{P}{c^{2}}+\rho\right) u_{i} u_{j}-P g_{i j}
$$

Where $P$ the pressure, $\rho$ density, $u_{i} u_{j}$ a four-vector, and $g_{i j}$ the metric tensor. The simplest case is the case of pure matter [4], where the equation reduces to:

$$
T_{i j}=\rho u_{i} u_{j}
$$

Which is nothing but the product of the pulse of each particle and flow of each particle:

$$
T_{i j}=m u_{i} \cdot n u_{j}=\rho u_{i} u_{j}
$$


Let us return to stress energy tensor of perfect fluids. This suggests these results:

$$
\begin{gathered}
T_{00}=\left(\frac{P}{c^{2}}+\rho\right) u_{0} u_{0}-P g_{00}=\left(\frac{P}{c^{2}}+\rho\right) c^{2}-P=\rho c^{2} \\
T_{0 i}=\left(\frac{P}{c^{2}}+\rho\right) u_{0} u_{i}-P g_{0 i}=0 \\
T_{i 0}=T_{0 i}=0 \\
T_{i i}=\left(\frac{P}{c^{2}}+\rho\right) u_{i} u_{i}-P g_{i i}=-P(-1)=P \\
T_{i j, i \neq j}=0
\end{gathered}
$$

What goes in line with the written matrix above.

The stress energy tensor, reduced to a perfect medium to describe the movement of the universe at very large scales (scales and stellar). It can be useful in studying superfluid environment.

\section{Part 3 - The stress energy tensor in Newtonian fluid.}

\section{Part A - First start: The article by T. Padmanabhan}

This article ${ }^{[5]}$ lays the foundation, in my humble opinion, the description of the field equations of Einstein hydrodynamic Navier Stokes. In this article, two methods used to obtain this equation.

The first method is to obtain extremization entropy associated with deformations zero surfaces. Here we do not explore this part because it opens the way to a thermodynamic description of Einstein's equations, which is not the subject of this article.

The second method, which is explained in this article, is to make the Navier Stokes equation in the context of a hypothetical fluid built on a normal zero. I will simplify explanations in order not to increase the current article and have a quick and general understanding of the contribution of T. Padmanabhan in the hydrodynamic equations Construction of Einstein fields.

It will therefore start to build a metric to normal $l$, with the coordinate $x^{3}$ which corresponds to a set of zero area, with $x^{0}=$ constant surface as well as a 2D surface as transverse coordinates $x^{A}\left(x^{1}, x^{2}\right)$. This gives the following metric:

$$
d s^{2}=q_{A B}\left(d x^{A}-v^{A} d t\right)\left(d x^{B}-v^{B} d t\right)
$$

This determines the extrinsic geometry of the null surface. I spend some computational details, and he comes to write these coefficients (called coefficients of Weingarten):

$$
\nabla_{\alpha} l \equiv X_{\alpha}^{\beta} \partial_{\beta}=X_{\alpha}^{\beta} \mathrm{e}_{\beta} ; \nabla_{\alpha} l^{\beta} \equiv X_{\alpha}^{\beta}
$$

It follows that the components of the matrix $3 \times 3$ of $X_{\alpha}^{\beta}$ can be expressed in the words $q_{A B}$ and $v^{A}$ allowing to achieve the following quantities:

$$
O_{A B}=-l_{m} \Gamma_{A B}^{m} \quad \omega_{0}=l^{m} \Gamma_{m 0}^{0} \quad \omega_{A}=l^{m} \Gamma_{m A}^{0}
$$


With variables, it deduces the new Navier Stokes:

$$
\left(\partial_{0}+v^{B} \partial_{\beta}\right)\left(-\frac{\omega_{A}}{8 \pi}\right)=\frac{1}{8 \pi} \partial_{\beta} \sigma_{A}^{B}-\frac{1}{16 \pi} \partial_{A} \theta-\partial_{A}\left(\frac{k}{8 \pi}\right)-T_{m A} l^{m}
$$

One then finds $-\omega_{A} / 8 \pi$ a density of movement, $k / 8 \pi$ a pressure, $\eta=1 / 16 \pi$ a shear viscosity coefficient (note also that the viscosity tensor can be write as follows $2 \eta \sigma_{B}^{A}+\xi \delta_{B}^{A} \theta$, with $\xi=-1 / 16 \pi$ an additional factor of 2 for the shear viscosity) and finally $T_{m A} l^{m}$ a external force.

In conclusion, the Einstein equations are identical to the Navier Stokes when they are projected on a zero surface.

important thing to clarify: The terms implicants derived from the viscous stress tensor, the derivative of the pressure and the time term external flows are zero in a free fall as they involve derivatives of the Christoffel symbol, the Due to the proportionality of these symbols to viscous stress and pressure.

In summary, observers find no dissipation but the force exerted on the viscous fluid does not disappear in the case of a free fall, which implies that this force has an existence independent of the observer.

I would like to mention the end of the article, which seems of paramount importance: "The relationship between the thermodynamics of backgrounds and dynamics of gravity began in the 70s as a provisional analogy. With the current results, it clearly reflects the underlying reality, that gravity is hydrodynamics."

\section{Part B - The theory of Franck Delplace.}

The stress energy tensor can be generalized in the fluid Newtonian framework. For this, we must rely on fluid mechanics ${ }^{[6]}$. The equation tensor of order 2 to define the mechanical behavior of a Newtonian liquid is as follows:

$$
t_{i j}=\eta \cdot \sigma_{i j}
$$

With $t_{i j}$ the stress tensor, $\eta$ represents the dynamic viscosity en Pas. $s$ and $\sigma_{i j}$ the shear rate tensor.

According to Franck Delplace, the stress energy tensor is simply a generalization of the stress tensor. If one cites his book "Porte Ouverte sur la Physique du $21^{\text {ème }}$ siècle" [6], he writes:

"This is not the mass of bodies that explains their behavior in the space-time continuum in which we are, but the stress or pressure they exert on the continuum."

Therefore, it follows that ${ }^{[7]}$ :

$$
T_{i j}=\eta \cdot \sigma_{i j}
$$

Starting from the Einstein equation, multiplies each side of the equation by the diffusivity pulse noted $D_{m}$ which it finally gets the left the product of the curvature diffusivity per pulse, which is none other than a gradient of speed :

$$
D_{m} R_{i j}=T_{i j}
$$

From there, Franck Delplace wrote that there is an equivalence between the laws of general relativity and Newtonian fluids mechanics. 
He then deduced a new constant:

$$
K=\frac{8 \pi G}{c^{4}} D_{m}
$$

Knowing that fluid mechanics kinematic viscosity noted $v$ and equal to $\eta / \rho$ it quickly deduce the following equality:

$$
K=\frac{\eta}{\rho} \frac{8 \pi G}{c^{4}}=\frac{1}{\eta}
$$

To finally define a relativistic viscosity, which is:

$$
\eta=\sqrt{\frac{c^{4} \rho}{8 \pi G}}
$$

This viscosity has the property of being extremely high. He then wrote a general property that is found in the book [6]:

"The space-time continuum is a viscous liquid of high dynamic viscosity."

However, just the problem of displacement in a photon in such a space-time. Indeed, if the viscosity is high, the photons would be forced to be slowed down, which would have the effect of creating an energy dissipation.

Franck Delplace away this problem by writing this property ${ }^{[6]}$ :

"The absolute void corresponds to the four-dimensional space-time Einstein which is a high viscosity liquid. It alone is not subject to thermal exchange of vibrational kind. It can undergo phase changes in the boundary conditions that are zero degrees Kelvin and high temperatures which appear plasmas."

The leads to the following hypothesis ${ }^{[6]}$ :

"A photon is a detached fragment of space-time continuum, extremely energy because matching an important vibrational state of this liquid."

In another article ${ }^{[7]}$ written in 2016, it more precise formulation of the Einstein equation. He comes to write this equality:

$$
G_{i j} v=k \sigma_{i j}
$$

This equation gives a relationship between Riemann curvature and velocity gradient. In fact, $\sigma_{i j}$ is the symmetric part of the velocity gradient tensor, which is also called strain tensor. From this equality, we can deduce this equation:

$$
G_{i j} v=\frac{8 \pi G v}{c^{4}} T_{i j}=k \sigma_{i j}
$$

Giving stress energy tensor :

$$
T_{i j}=\frac{c^{4} k}{8 \pi G v} \sigma_{i j}
$$


One can easily notice that:

$$
\eta=\frac{c^{4} k}{8 \pi G v}
$$

Which brings us to the following equation:

$$
T_{i j}=\eta \cdot \sigma_{i j}
$$

We find a tie between stress tensor and noted $T_{i j}$ the strain tensor rate $\sigma_{i j}$. The amount $\eta$ would represent the dynamic viscosity of the theory of general relativity.

This equation leads him to conclude that the planets move in curved space-time of Einstein is due to the existence of a nonzero value of dynamic viscosity of spacetime.

\section{Part C - The theory of Stefano Liberati and Luca Maccione.}

Stefano Liberati (SISSA) and Luca Maccione (University of Munich) proposes that space-time is a form of superfluid structure, based on space-time in quantum gravity ${ }^{[9]}$. Indeed, it is postulated that the Planck scale, ie $10^{\wedge}-33 \mathrm{~cm}$, spacetime takes either a continuous structure but discreet, as if it were composed of a multitude "atom" or "elementary grains" assembled together. Contrary to general relativity, which describes the behavior of the space-time globally, quantum gravity models attempt to describe the composition of this space-time; for Liberati and Maccione, space-time would, like water which is composed of molecules of $\mathrm{H} 2 \mathrm{O}$, a fluid whose components are still unknown.

To do this, they first describe a viscous wave equation for velocity perturbations $v^{\mu}=\nabla^{\mu} \varphi$ of the following form:

$$
\partial_{t}^{2} \varphi_{1}=c^{2} \nabla^{2} \varphi_{1}+\frac{4}{3} v \partial_{t} \nabla^{2} \varphi_{1}
$$

I spend the computational details are not of paramount importance for the analysis we will do it.

The scientific team then made the following remark: "On the adoption of hydrodynamics as large-scale model of a space emerge, it is quite interesting to keep in mind that the dissipation discussed above appears in a gradient expansion such as a first order correction to the equations of an ideal fluid."

The above equation can be described by the generalization of the Navier Stokes:

$$
\partial_{t}^{2} \varphi_{1}=c^{2} \nabla^{2} \varphi_{1}+\sum_{n=2}^{\infty} \frac{4}{3} v \partial_{t} \nabla^{n} \varphi_{1}
$$

They believe (I pass the computational details) that the energy loss rate (energy dissipation) can then be written by the formula:

$$
\sigma_{2} c^{2} \frac{k^{3}}{M_{P l}}=2 \omega \Gamma \quad \text { avec } \quad \sigma_{2}=\frac{4 v_{2} M_{P l}}{3 c} \text { et } \Gamma \approx \frac{\sigma_{2} k^{2}}{2 M_{P l}}
$$


Where found $M_{P l}$ the Planck mass, $k$ a energy, $\sigma$ the dimensionless coefficient controlling the extent of the violation of Lorentz and $\Gamma$ the energy loss rate. By calculating $\sigma_{2}$ the photon ultra relativistic frame $80 \mathrm{TeV}$ from the Crab Nebula, with the distance to the Crab Nebula, which is $D_{c r a b}=1,9 k p c$, we have:

$$
\sigma_{2} \leq \frac{2 c h}{D_{c r a b}(80 \mathrm{TeV})^{2}}=1,3 \times 10^{-26}
$$

They then observe that the photons are produced by diffusion inverse Compton electron and positron accelerating and propagating in the nebula. This constraint can then be applied to the same lepton in assume they have the same energy as photons produced.

They then deduce that for neutrino example, the stress is on the order of $2 \times 10^{-27}$.

Researchers have shown and the viscosity of the fluid will tend to dissipate quickly photons along their route. Now we can see the path of photons emitted by bodies located several light years.

In conclusion, writing hydrodynamics as a general framework of a space-time emerging below the Planck energy, they come to the results obtained in the limit order rained down (they call viscosity of space-time) are very tight, dissipative effects beyond the Planck scale. Finally, they conclude that space-time should be emerging a superfluid.

The modeling of space-time as a fluid has been theorized in the past, but the proposed assumptions take into account the potential effects of fluid viscosity on the photons, ie the speed variations they would suffer in terms of their energy. The model Liberati and Maccione takes into account all other effects, including the effects of dissipation, making them more comprehensive theoretical model.

Thereby, the theory and Stefano Liberati Luca Maccione can only work in the context of a transformation of the stress energy tensor stress tensor Newtonian fluids.

\section{Part 4 - The space-time, a non-Newtonian fluid.}

Luis Lehner, Huan Yang and Aaron Zimmerman also began to theorize that space-time could suffer the same turbulence that fluid ${ }^{[10]}$ : confining a black hole in an anti de Sitter space, turbulence effects are appeared. Therefore, the viscosity around a black hole in rapid rotation is smaller than around it, suggesting that the viscosity is influenced by the curvature of the space-time.

First, they took as a starting point the Reynolds number. This lets you know when fluid becomes turbulent, especially by the following equation:

$$
R e=\frac{\rho}{\eta v \lambda}
$$

With $\rho$ the fluid density, $\eta$ fluid viscosity, $\lambda$ a wavelength and $v$ the fluid velocity. It turns out that the medium-gravity duality indicates that there are relativistic hydrodynamic disturbances (given by the equation of state $p=\rho / d$ ). This fact is observable in the case of black holes.

To validate this result in a more realistic setting, the researchers studied black holes ultra-fast rotation. Indeed, fluid mechanics applied to black holes describes spacetime around such black holes as very low viscosity, and therefore subject to higher turbulence. 
I will not detail here the calculations, everything is explained in ${ }^{[10]}$. I would note just a formula that seems important. In the context of a 4-dimensional space with a stationary black hole defined by its mass and spin yields the following frequency:

$$
\omega_{l m n} \approx \frac{m}{2}-\frac{\delta \sqrt{\varepsilon}}{\sqrt{2}}-i\left(n+\frac{1}{2}\right) \frac{\sqrt{\varepsilon}}{\sqrt{2}}
$$

With $\sqrt{\varepsilon}$ a rate, $l, m, n$ which are the angular numbers respectively, and azimuthal harmonics, and a function $\delta$ of $l, m, n$. Following this formula, they deduce that a decrease of almost normal modes (mode power dissipation of an object or a disturbed field) involves a low viscosity and an increase in the Reynolds number, so turbulence higher.

therefore They then applied a model of non-linear disturbances to these black holes and the simulation result was uplifting: space-time has become turbulent, just as in a fluid subjected to stir. If this result was never achieved before is that no non-linear study of a gravitational system had been undertaken (because extremely complex), while the turbulence phenomenon is non-linear in nature. It is precisely because of this complexity that I will not detail here the calculations.

\section{Part 5 - Conclusion.}

We saw in the first part, the stress energy tensor, in general relativity, is that as the sole function of defining the energy density of the corpse that causes the curvature of space-time. However, we noticed that the stress energy tensor used in general relativity was other than a generalization of the stress tensor used in fluid mechanics. Thus, the interpretation that it is the pressure of the corpse which causes the curvature of the space-time is quite plausible.

The stress energy tensor, reduced to a perfect fluid, we can better understand the stress energy tensor of general relativity: in fact, it consists mainly in its matrix under pressure and energy density, which confirms our interpretation that it is the pressure of the body which are the source of the curvature of the spacetime.

The use of stress energy tensor for a perfect fluid is handy when it comes to studying the infinitely large, to stellar levels, galaxy and beyond. Indeed, the space-time behaves on this scale as a perfect fluid.

Just writing the Newtonian fluid stress energy tensor. In this case, the stress energy tensor becomes a fluid having a viscosity.

The equation that defines the evolution of a Newtonian fluid and Navier-Stokes. T. Padmanabhan will then change the Einstein field equations, Navier Stokes equation under zero area. He then deduced that gravity is hydrodynamics.

Franck Delplace leave in the same vein. It started from the idea that the stress energy tensor is none other than the stress tensor of the fluid mechanics but extended to general relativity. He then deduces that there is equality between the stress energy tensor viscosity and velocity gradient. He was the first to define, from only equations Planck and the basics of fluid mechanics and without using the Navier Stokes, a hydrodynamic equation, a Newtonian fluid, the Einstein equation.

Finally, Stefano Liberati and Maccione Luca will be the first to find that space-time, at the quantum scale, would be a superfluid. This finding their came from an analogy in a fluid-universe, the particles would travel in space-time as waves on the surface of the water. From there, they thought that general relativity could be built from fluid mechanics. In such cases, there must be power dissipation and speed variation. To confirm their ideas, they thought that the photons of high energy that travel great distence lose a significant fraction of their energy. Thus, they observed the Crab Nebula, which should issue such photons. However, nothing was observed confirming their theories. 
I ranked these three theories in Newtonian fluids as a Newtonian fluid to the property of being independent of the mechanical stress is applied to it. In other words, the viscosity is constant. Thus, this is the time-space and its viscous property that has a direct impact on the movement of bodies.

Finally, the description of the stress energy tensor as a non-Newtonian fluid is similar to the theory, described by Luis Lehner, Huan Yang and Aaron Zimmerman. According to this theory, when confines a black hole in an anti Sitter space, turbulence is observed, these can be described by fluid mechanics. Moreover, the smaller the viscosity, the greater the turbulance. It turns out that at the level of black holes ultra rapid rotation, the viscosity is very low. This theory is therefore a theory characterizing the space-time as a non-Newtonian fluid.

An important note is to do. Whether in the Luis Lehner theory Huan Yang and Aaron Zimmerman and that of Stefano Liberati and Luca Maccione, no changes were made to the stress energy tensor. However, the fact that time-space or a fluid is directly related to the stress energy tensor, since it is the latter which affects the curvature of space-time, and therefore the viscosity.

Describe the Einstein equations as fluid is anything new in science, even if it has already been thought in the 70s by studying more in depth the links that connect fluid mechanics, physics and general relativity, perhaps finally discover us the nature of spacetime and quantum gravity.

\section{Part 6 - Bibliography.}

[1] Eric Gourgoulhon (2013-2014), Relativité générale, CNRS, Observatoire de Paris

[2] Science.ch (2017), 50. Relativité générale, Cosmologie

[3] Richard Taillet (2013), Equation d'Einstein, Podcast de l'université de Grenoble, Relativité générale, épisode 11

[4] André Lichnebrowicz (1966), Etude mathématique des fluides thermodynamiques relativistes, collège de France, Commun. Math. Phys. 1, 328-373

[5] T. Padmanabhan (2011), The Hydrodynamics of Atoms of Spacetime: Gravitational Field Equation is Navier-Stockes Equation, International Journal of Modern Physics, ArXiv:1012.0119v1

[6] Franck Delplace (2009), Porte ouverte sur la physique du $21^{\text {ème }}$ siècle, éditeur Franck Delplace

[7] Franck Delplace (2014), Liquid Spacetime (Aether) Viscosity, a way to Uniy Physics, gs.journal

[8] Franck Delplace (2016), Reynolds Number and Spacetime Curvature, Fluid Mechanics Open Access, DOI:10.4172/fmoa.1000125

[9] Stefano Liberati (SISSA) and Luca Maccione (2010), Astrophysical Constraints on Planck Scale Dissipative Phenomena, Physical Review Letters, ArXiv:1309.7296

[10] Luis Lehner, Huan Yang et Aaron Zimmerman (2014), Turbulent Black Holes, arXiv $1402.4859 \mathrm{v} 2$ 